\title{
Les mots du journalisme : de la terminologie à la culture professionnelle
}

Journalism in its own words: from terminology to professional culture

\section{Caroline Peynaud}

\section{(2) OpenEdition}

1 Journals

Édition électronique

URL : http://journals.openedition.org/asp/4690

DOI : 10.4000/asp.4690

ISSN : 2108-6354

Éditeur

Groupe d'étude et de recherche en anglais de spécialité

\section{Édition imprimée}

Date de publication : 23 octobre 2015

Pagination : 25-44

ISSN : 1246-8185

\section{Référence électronique}

Caroline Peynaud, «Les mots du journalisme : de la terminologie à la culture professionnelle », ASp [En ligne], 68 | 2015, mis en ligne le 01 novembre 2016, consulté le 02 novembre 2020. URL : http:// journals.openedition.org/asp/4690; DOI : https://doi.org/10.4000/asp.4690

Ce document a été généré automatiquement le 2 novembre 2020.

Tous droits réservés 


\title{
Les mots du journalisme : de la terminologie à la culture professionnelle
}

Journalism in its own words: from terminology to professional culture

\author{
Caroline Peynaud
}

\section{Introduction}

1 En tant que dénominations des concepts qui structurent un domaine, les termes employés par les professionnels représentent, pour le chercheur, une porte d'entrée vers leur culture. La notion de culture professionnelle est entendue ici au sens de l'ensemble des valeurs, exprimées à travers les héros, rituels et symboles, qui régissent les pratiques dans une profession donnée (Hofstede 2005 : 7). Le rôle des termes dans la culture est défini dans le cadre de l'étude des variétés spécialisées de l'anglais, définie comme suit par Catherine Resche :

Since any specialized discipline or professional field is based on theory or practice that are expressed through language, language can serve as a starting point for further investigation into specific domains and specialized communities, and into their culture and discourse [...] terms convey much more than the concepts they denote. (2013: 14)

Ainsi, les termes constituent un point d'entrée privilégié pour caractériser la culture d'un milieu professionnel tel que celui des journalistes de presse écrite aux États-Unis. L'étude d'une variété spécialisée de l'anglais passe par la connaissance des caractéristiques culturelles du domaine auquel elle se rattache autant que par celle des discours produits par ses acteurs (ibidem: 13). Les termes sont ancrés dans la culture, c'est pourquoi le travail du chercheur, lorsqu'il étudie un domaine spécialisé, implique de comprendre les acteurs, les communautés auxquelles ils appartiennent, leurs origines, leur histoire et leurs conventions (ibid.: 14). Dans cette optique, c'est le 
discours interne qui est au centre de cette étude, c'est-à-dire le discours produit par des professionnels à destination d'autres professionnels.

L'approche par les termes du discours interne permet de s'écarter d'un angle purement culturel qui peut parfois donner lieu à des représentations contradictoires et qui ne permet pas toujours de mettre en évidence les aspects spécialisés propres à certains milieux professionnels. En effet, il est difficile d'identifier ce qui est spécialisé dans le domaine du journalisme aux États-Unis, pour plusieurs raisons qui sont développées dans les sections suivantes. L'analyse des termes permet de faire émerger une meilleure représentation des schémas culturels propres à ce domaine et d'éclairer certains aspects culturels de cette profession ${ }^{1}$.

\section{Termes et culture dans le domaine journalistique}

\subsection{Termes, terminologie et culture}

4 En tant que dénomination de concepts, les termes révèlent la structuration conceptuelle du domaine terminologique auquel ils appartiennent. Maria Teresa Cabré souligne que " pour exister en tant que terme, une forme linguistique doit désigner un concept appartenant à un domaine et déterminé par une définition » $(1998: 147)$. Trois éléments fondamentaux apparaissent ici. En premier lieu, la primauté du concept sur sa désignation dans l'étude des termes. En outre, le domaine dans lequel se place le terme est essentiel à sa compréhension. Au sein de son domaine terminologique, le concept est mis en relation avec les autres concepts du domaine qui permettent de le comprendre alors que, hors du domaine, le terme peut n'être qu'une simple unité lexicale faisant partie du vocabulaire général, comme le remarquent Ruan C. Sager et al.: " the lexical level of special languages must be defined as including all items which have special references, regardless of whether they have general reference or not " $(1980: 230)$. Enfin, le terme doit avoir une définition, ce qui signale l'importance des dictionnaires terminologiques.

5 Ainsi, un terme est avant tout employé pour exprimer des connaissances spécialisées. C'est en ce sens qu'il vise la précision de la communication, voire la biunivocité, définie par Eugen Wüster comme la stabilité de la relation entre dénomination et concept, laquelle devrait idéalement interdire l'homonymie, la polysémie ou la synonymie (Candel 2004: 17), même si E. Wüster lui-même reconnaissait que ce principe était un "vœu pieux» (ibid.: 29). R. Sager et al. formulent le même principe: "The special lexicographer or terminographer makes his decision about the unity of a term with reference to the knowledge structure of a discipline" (1980: 233). Un terme doit représenter précisément un concept et c'est en cela que les termes peuvent être considérés comme les indices principaux de la structuration conceptuelle d'un domaine spécialisé.

Puisqu'un terme exprime des connaissances spécialisées, ceux qui sont employés par les acteurs d'un domaine reflètent la manière dont ils se représentent leur métier. C'est en ce sens qu'ils représentent une porte d'entrée vers la culture professionnelle, ainsi qu'un aperçu de l'évolution historique de cette culture : «It is most important to be able to trace terms to their creators and not to cut them off from their historical roots and socioterminologists insist on this point too" (Resche 2013 : 96). Dans la même optique, la persistance de certains termes dans l'histoire reflète sans doute une certaine stabilité de la culture professionnelle à travers le temps. 
7 L'approche adoptée ici diffère cependant de celle qui a été préconisée en terminologie pour l'étude d'un domaine, dans la mesure où la présente étude ne considère pas les termes comme la seule voie d'accès a la culture des journalistes aux États-Unis. Une étude précédente, fondée sur des entretiens et des questionnaires (Peynaud 2013: 70), avaient permis de déterminer certains aspects de cette culture et, surtout, de révéler plusieurs contradictions dans la représentation que les journalistes donnent d'euxmêmes. Cette étude avaient déjà conduit à relever plusieurs termes employés dans le domaine.

8 Or, dans le contexte de réponses à un questionnaire ou d'entretiens conduits par un chercheur extérieur à la profession, les journalistes produisent un discours de représentation, dans lequel ils tentent de maîtriser l'image qu'ils donnent d'eux-mêmes à l'extérieur. L'intérêt majeur d'une approche par les termes du discours interne réside dans le fait que, dans ce type de discours, les journalistes ne se placent pas dans une posture de représentation vis-à-vis de l'extérieur : ils communiquent entre eux, ce qui conduit à la production d'un discours probablement plus proche de leurs pratiques professionnelles, et cela même si les articles sélectionnés pour le corpus sont accessibles au grand public. Cependant, il est avant tout essentiel de comprendre certaines caractéristiques culturelles du domaine avant de nous concentrer sur les termes.

\subsection{Fondamentaux du domaine du journalisme aux États-Unis}

9 En premier lieu, lorsque nous parlons du domaine du journalisme, c'est en référence à la notion de domaine spécialisé telle qu'elle a été théorisée par Michel Petit :

Nous appellerons domaine spécialisé tout secteur de la société constitué autour et en vue de l'exercice d'une activité principale qui, par sa nature, sa finalité et ses modalités particulières ainsi que par les compétences particulières qu'elle met en jeu chez ses acteurs, définit la place reconnaissable de ce secteur au sein de la société et d'un ensemble de ses autres secteurs et détermine sa composition et son organisation spécifiques. (Petit 2010 :9)

Nous envisageons la culture du domaine en tant que culture spécialisée, qui produit un discours répondant à cette spécialisation. Cette section définit plus précisément les difficultés qui émergent de l'étude de ce domaine spécialisé.

Le premier obstacle à la caractérisation du domaine est la difficulté de définir ses limites, de déterminer qui est journaliste et qui ne l'est pas. Du point de vue constitutionnel, aux États-Unis, il ne peut pas y avoir de différence entre un journaliste professionnel et un citoyen non professionnel publiant des informations, sur un blogue, par exemple. C'est du moins de cette manière que le Premier Amendement a été le plus souvent interprété par la Cour Suprême, interdisant de fait toute législation portant sur le journalisme ou toute réglementation spécifique (Watson 2008 : 196), interdiction qui pourrait conduire à dénier aux journalistes américains tout statut professionnel, du moins sur un plan strictement juridique. Dans ces conditions, il est difficile d'identifier précisément les acteurs du domaine, ce qui pose problème pour définir son caractère spécialisé. Cette vision est partagée par les journalistes que nous avons eu l'occasion d'interroger, pour qui, en général, un blogueur est journaliste au même titre qu'un employé du New York Times, même si, de l'aveu des derniers, il existe tout de même quelques différences en matière de pratiques. En effet, certains journalistes nous ont expliqué qu'un blogueur pouvait exercer le même métier qu'un employé du New York 
Times tandis que deux professionnels du même journal pouvaient pratiquer deux types de journalisme tout à fait différents. Cependant, aucun des professionnels interrogés n'a été en mesure de définir précisément ce qui différenciait ces pratiques (Peynaud 2013 : 246). Ainsi, la définition du journalisme en général, et en particulier des types de journalisme qui le composent, est assez floue aux États-Unis. Cette première difficulté est à la source d'autres contradictions relevées dans le discours des professionnels.

Ainsi, les journalistes sont très attachés à l'image traditionnelle du reporter solitaire, attentifs à représenter leurs structures professionnelles comme très peu hiérarchisées et leur travail comme individuel et unique (Padioleau $1985:$ 168). Or, cette idée ne résiste pas à l'analyse des pratiques dans l'entreprise, où les rôles de chacun sont clairement définis et où l'article est l'œuvre non pas d'un individu, mais d'une chaîne d'individus qui conduit à la publication finale d'un discours qui peut être qualifié d'« institutionnel» (De Ceglie 2007 : 198).

Enfin, les journalistes soulignent constamment leur mission au service de l'intérêt général bien que, de leur propre aveu, celle-ci se trouve parfois en contradiction avec les enjeux économiques auxquels les publications sont soumises. Les valeurs et l'éthique semblent malgré tout tenir une grande place dans le discours des professionnels, ce qui peut être dû au discours de représentation qui a dominé lors des entretiens, comme cela a été expliqué plus tôt.

Il apparait dans ces premières remarques que l'image du journalisme est faite de contradictions. Nous cherchons à comprendre les conceptions du métier que les termes employés dans le discours interne reflètent. Étant donné l'image de faible spécialisation que suggèrent nos précédents travaux, le domaine semble peu structuré. L'objectif de cette étude est de comprendre s'il dispose néanmoins d'une structuration conceptuelle véhiculée par les termes.

\subsection{Sources}

Pour le comprendre, deux revues de communication interne ont été étudiées. Le choix de ce type de publications a été motivé par la nécessité de prendre en compte un discours dont à la fois les locuteurs et les destinataires sont des professionnels du domaine. Cependant, nous avons également fait le choix d'un nombre réduit de revues et d'articles dans la mesure où l'objectif de cette étude n'est pas de réaliser une analyse quantitative, mais avant tout de proposer une première approche des termes employés pour décrire la profession.

La première revue est American Journalism Review, publiée par Philip Merrill College of Journalism à l'Université du Maryland. La mission de cette revue est définie comme suit: "AJR covers the news media, with a focus on innovation, entrepreneurship and digital storytelling " («About », American Journalism Review). La seconde, plus concentrée sur les développements économiques du secteur, est Editor and Publisher, dont la mission est décrite ainsi :

Editor \& Publisher is the authoritative journal covering all aspects of the newspaper industry, including business, newsroom, advertising, circulation, marketing, technology, online and syndicates. (" About ", Editor and Publisher) les décisions dans le journal, les rédacteurs en chef et les éditeurs, tandis qu'American Journalism Review s'adresse plus généralement à tous les journalistes. 

avons considéré comme terme toute unité lexicale dont le sens est attesté dans le domaine du journalisme ou des sciences de l'information dans Le Grand Dictionnaire Terminologique ${ }^{3}$. Une recherche sur l'origine historique des termes a également été conduite, ainsi que sur la période à partir de laquelle ils sont attestés dans le métier, grâce à un dictionnaire étymologique en ligne (etymonline.com ${ }^{4}$ ). Toutes les informations concernant les premières attestations des mots et termes cités ainsi que leurs définitions en sont tirées. Certaines unités lexicales courantes ont été analysées à l'aide du même outil, même si elles ne peuvent pas être décrites comme des termes, dans la mesure où elles ajoutent parfois des informations importantes à la compréhension de la structuration du domaine.

\section{Les termes du domaine}

\subsection{Panorama des termes relevés}

21 Dans un premier temps, un panorama général des termes relevés dans les articles conduit à remarquer que plusieurs sous-domaines de la profession sont décrits à l'aide de termes, ce qui met en évidence une certaine structuration conceptuelle du domaine. Ces sous-domaines sont les suivants :

22 - l'édition et l'imprimerie : a banner headline, a banner, subheads, publishing, prepress, shafted presses, distribution footprint, distribution frequency. Il faut par ailleurs remarquer que plusieurs termes issus du lexique de l'imprimerie sont employés dans d'autres sous-domaines, comme editor, ce qui souligne l'importance de ce sous-domaine dans la profession.

23 - les métiers du journalisme : editor, copy editor, editor in chief, auxquels nous pouvons ajouter journalist et reporter qui, s'ils font partie du vocabulaire courant, font l'objet d'une distinction particulière dans les articles, comme il est montré dans une section ultérieure. Des rôles plus précis font partie du même sous-domaine tels que city editor ou investigative reporter.

24 - les genres de la presse, sous-domaine particulièrement prolifique : column, editorial, feature, leader, profile, op-ed, etc. 


\section{choisis pour décrire l'activité des journalistes. L'un des verbes les plus courants dans le} corpus est run, dont les emplois sont très variés :

(1) «[...] like The New York Times, which is currently running a series called 'Couch'»

(AJR3)

(2) « the New Republic story runs more than 6,000 words » (AJR4)

(3) « For every 'Print isn't dead' headline newspapers run, there's at least five 'So-

and-so newspaper reports declining revenue, numerous layoffs'. (EP2)

(4) « like a popular biweekly bar and club guide, Nightspots, which has a run of 4,000 copies. » (EP10)

31

- l'éthique et les pratiques : well-sourced, watchdog, leak, scoop, newsworthy, off-therecord, etc. media publishing, multi-platform publishing, digital journalism, click-based metrics, etc. relevés, les sections suivantes présentent une analyse plus détaillée des termes et des quils entretiennent entre eux. Elle permet néanmoins de constater que les a des sous-domaines qui structurent le domaine journalistique, ce qui indique qu'il existe bien une terminologie interne journalistique.

décrive précisément l'activité journalistique en elle-même. Au contraire, les lexèmes employés pour se référer à l'activité journalistique sont bien présents dans les textes du interne. Par exemple, le journaliste est plus souvent appelé author (l'expression authoring a piece est relevée à plusieurs reprises) ou writer que journalist ou reporter, niant en quelque sorte la spécificité du métier. D'autres termes viennent appuyer cette idée. Par exemple, lorsqu'un rédacteur donne une tâche à un journaliste, il emploie le terme d'assignment et lorsqu'une histoire est inventée, le mot choisi est fabricated. Or, ce lexique n'est pas spécifique au domaine journalistique, alors qu'il décrit des notions qui lui sont centrales. employés, lorsque ceux-ci expriment clairement l'action entreprise. Cependant, il est remarquable que la plupart des mots qui décrivent l'activité journalistique fassent affichée, ici, le discours est très peu technique, comme si cette technicité était rejetée de la description de l'activité. Cette observation confirme que les limites du domaine du journalisme sont floues aux États-Unis, comme souligné plus tôt: il semble que les journalistes choisissent, pour des raisons, peut-on supposer, de cohérence avec l'image de la profession à laquelle ils adhèrent, d'employer un discours très peu technique.

a variation sémantique du verbe run est remarquable : il est employé ici dans le sens de publishing (1), pour exprimer la longueur d'un texte (2), la publication d'un titre (3), ou encore la diffusion d'un magazine (4). Alors que des termes plus précis et plus 
techniques existent pour exprimer ces idées, les professionnels qui rédigent ces textes choisissent d'employer un mot plus courant. Par exemple, nous relevons à plusieurs reprises les expressions make the frontpage, ou encore fix a mistake, là où correct serait plus précis et attesté dans le domaine pour remplacer fix, ou be published à la place de make. Ces observations conduisent à penser que l'emploi de mots peu spécialisés relève d'un choix, conscient ou inconscient, des auteurs.

Une première observation des termes permet de comprendre qu'ils reflètent la conception peu spécialisée du journalisme précédemment soulignée. Seuls certains sous-domaines de la profession semblent en effet employer des termes spécifiques, ce qui souligne une fois de plus le fait que la question de la spécialisation pose problème dans cette profession. Dans les sections suivantes, nous cherchons à comprendre pourquoi certains sous-domaines semblent plus structurés que d'autres, ce qui peut s'expliquer en partie par l'évolution historique de cette profession.

\subsection{Les premiers journaux et les printer-journalists}

33 Les premiers journaux apparaissent à la fin du dix-septième siècle et évoluent sur le même modèle pendant plus d'un siècle. Ce modèle est celui de journaux partisans, financés principalement par les partis politiques et les abonnements, peu nombreux. La presse a alors une diffusion très limitée, parmi l'élite du pays. Par ailleurs, c'est l'époque des printer-journalists, où ceux qui impriment les journaux sont d'abord des imprimeurs possédant une presse et sachant la faire fonctionner. Pour la plupart, la publication d'un journal n'est qu'une activité annexe par rapport à l'impression de documents pour lesquels ils sont rémunérés. Ces journalistes imprimeurs cumulent les activités de collecte de l'information, de mise en forme, d'impression et de distribution. La définition du métier est assez vague, mais certains termes et lexèmes sont pourtant déjà bien établis, principalement en rapport avec le domaine de l'imprimerie.

Le mot de newspaper est attesté dès 1660. Il est autant employé dans le corpus que sa forme tronquée, paper, même si la dimension d'objet en papier est remise en cause dans plusieurs articles. L'un des textes signale notamment « news is not outdated, but the word paper is » (EP2) tandis qu'un autre explique :

That's one of the things that really frustrates me: that everybody says, 'Oh, newspapers are dead!' But that's not true. It may be a case of fewer people looking at print, but that's not the only place where we do journalism anymore. (EP3)

Malgré la présence du mot paper dans ce nom, newspaper semble en venir à désigner bien plus que des publications papier.

De plus, les protagonistes de la publication d'un journal sont déjà clairement identifiés. Si le terme de printer-journalist est une invention des historiens, on parle de journalist dès 1690 , en tant que celui qui publie un journal, au sens, à l'époque, d'une publication quotidienne. Le mot reporter est un peu plus récent, attesté dès 1798, après la Révolution. L'évolution de journalist à reporter est intéressante, dans la mesure où le premier fait référence à l'action de publier, tandis que le second se rapporte à l'activité journalistique, celle de rechercher l'information et d'en faire état dans une publication, en tant que dérivé du verbe report. Dans les usages actuels, les deux termes sont employés de manière distincte : journalist recouvre toutes les personnes travaillant à la production dans un journal, ce qui inclut les rédacteurs en chef ou les correspondants, tandis que reporter est un hyponyme de journalist qui ne désigne que ceux qui 
recherchent l'information. Dans les articles du corpus, reporter est principalement employé pour faire référence à une personne en particulier, qui se définit ou est définie comme reporter, souvent en lien avec une rubrique, par exemple : " 'that it is perfect' said Ravi Somaiya, media reporter for The New York Times» (AJR10) ou "In 1973, investigative reporter Lucy Morgan was sentenced to eight months in jail [...]» (EP7). Les noms de métiers tels que media reporter ou investigative reporter, qui réfèrent à des concepts clairement définissables dans la profession, peuvent être considérés comme des termes.

37 À l'inverse, journalist est davantage employé de manière abstraite, pour se référer au rôle général: "the jury's question seemed to insinuate that the prosecution of a journalist was a direct violation of the First Amendment » (EP7). Individuellement, les professionnels semblent d'abord se définir par leur activité, le fait de "rapporter » l'actualité. Si ces mots ne sont pas des termes spécialisés, puisqu'ils sont passés dans le langage courant, il est néanmoins intéressant de remarquer que les rôles principaux et les fondamentaux de l'activité sont déjà bien établis à cette époque. Il en est de même pour les termes de source, correspondent ou editor, qui datent de la même période. Le premier est d'ailleurs source de nombreux dérivés tels que le verbe source (an article) ou l'adjectif well-sourced.

38 Ainsi, par dérivation, l'activité principale réalisée par les journalistes est le reporting, terme qui suggère une activité de recherche et un récit objectif des événements. Le corpus contient vingt-cinq occurrences du terme reporting, comme dans cet exemple où cette activité est clairement identifiée comme définitoire du métier :

Jamie Lee Curtis Taete, Vice's west coast editor, contacted by AJR, said he did not feel he was qualified to weigh in on narratives in journalism because "the majority is experiential writing and doesn't really include much actual reporting." "I would say I'm more of a 'blogger' than a journalist," he added, in an email. (AJR2)

Le métier de journaliste se définit d'abord par l'activité de reporting, le reporter semblant être l'essence du journaliste.

Editor est également un terme très courant dans les articles du corpus, désignant tout responsable hiérarchique : responsable d'une rubrique (city editor, commentary editor...), d'une aire géographique (West coast editor) ou d'une publication (Buzzfeed editor, Eagle Tribune managing editor, editor-in-chief ou chief editor). Ce terme est également employé pour les journalistes qui n'écrivent pas directement les articles : on parle ainsi de copy editor pour les correcteurs ou de standards and practices editor pour désigner le référent en charge des questions éthiques. En résumé, le terme editor désigne, avec une certaine plasticité sémantique, tous ceux qui sont impliqués dans la production du contenu sans être de simples reporters. Les editors ont systématiquement un rôle de vérification et de correction des articles avant leur publication, ce qui est cohérent avec le verbe edit, dont le nom est dérivé. Ce dernier ayant trait à l'imprimerie, il n'est pas étonnant qu'il soit apparu dans le domaine dès l'époque des printer-journalists. C'est pourquoi il est surprenant de remarquer que l'adjectif editorial en est venu à désigner le contenu informationnel d'un journal: the editorial side est alors opposé à the advertising side, opposant les journalistes aux non-editorial people (EP5).

41 Enfin, un autre terme de cette époque est issu du vocabulaire de l'imprimerie, c'est celui de column. Le premier sens de ce terme, celui d'un texte publié dans un journal, date de 1785. Cependant, si le terme existe toujours aujourd'hui, son sens a évolué : dans le journalisme moderne, il désigne des articles d'opinion qui sont publiés de manière régulière (ce sens n'est attesté qu'en 1920); le métier de columnist en est 
dérivé. Il n'est pas surprenant que seul le sens d'article d'opinion ait perduré aujourd'hui : dans la mesure où la presse du dix-huitième siècle était partisane, ce qui était appelé column exprimait nécessairement une opinion. C'est justement en rupture avec cette époque que le terme de column en est venu à désigner une partie très réduite de ce qui était publié, tandis que de nouveaux genres étaient créés, pour répondre à une nouvelle conception de l'information d'actualité.

\subsection{La période de la presse à un penny}

Au début du dix-neuvième siècle, un nouveau type de presse émerge, appelée la presse à un penny (penny press). En rupture avec la presse partisane à petite diffusion, qui tirait ses revenus des partis politiques et des abonnements, la presse à un penny tire la plus grande part de ses revenus de la publicité. Or, pour que les annonceurs investissent dans les journaux, ceux-ci doivent être diffusés à grande échelle. C'est donc d'abord pour des raisons économiques que cette presse abandonne le soutien partisan pour souscrire à la valeur d'objectivité, afin de se donner une chance de plaire au plus grand nombre (Schudson 1990 : 163). C'est également le moment où le journalisme se constitue comme métier rémunéré, structuré par les premières associations professionnelles, même si celles-ci, n'ayant qu'un rôle consultatif, ne sont pas déterminantes pour identifier les professionnels du journalisme.

Dans un contexte où les enjeux économiques deviennent décisifs, plusieurs termes reflétant l'urgence de l'information font leur apparition, par exemple leak (1832), tip (1845) ou scoop (1874). Le dernier terme est indiqué comme faisant partie de l'argot commercial dans le sens de "appropriate so as to exclude competitors". Les emprunts à l'argot sont en effet assez courants dans le domaine, ce qui témoigne probablement de la volonté d'employer ce langage relâché, peu technique, mentionné plus tôt.

Ces termes sont particulièrement remarquables par le nombre des dérivés auxquels ils ont donné naissance. Par exemple, leak n'est attesté dans le domaine qu'en tant que verbe signifiant " come to be known in spite of efforts at concealment ». Cependant, dans le corpus, nous le trouvons également sous forme de nom (a leak) ou de nom d'agent (leaker). De la même manière, tip existe aussi sous forme de verbe et de nom d'agent, et il en est de même pour scoop, employé régulièrement au passif (being scooped) avec le sens de se faire devancer par un autre journaliste dans la collecte d'information.

En plus de la description de l'activité, de nombreux termes désignant les genres journalistiques sont créés à cette période, tels que editorial, feature ou leader. Il semble que ce soit à cette époque que s'opère la séparation entre articles d'opinion et articles objectifs ${ }^{5}$. Dérivé d'editor, editorial désigne un article d'opinion écrit par un editor tandis que le leader est l'editorial principal d'un journal. Feature signifie un article objectif écrit dans une forme longue. Cette division entre faits et opinion est cohérente avec l'importance nouvellement accordée à la valeur d'objectivité. C'est cette distinction fondamentale qui détermine la création de nombre d'autres genres dans les périodes qui suivent.

Dans la lignée de l'apparition de la valeur d'objectivité, le journalisme se conçoit davantage comme un contre-pouvoir, ce qui donne le contexte de l'apparition de la notion de watchdog (1845), type de journalisme dont le rôle est de surveiller le gouvernement ou les pouvoirs économiques et de s'assurer qu'ils fonctionnent de manière honnête et transparente. Or, dans les articles du corpus, de manière 
surprenante, ce terme est uniquement employé pour parler de surveillance des médias eux-mêmes, par exemple: "there are staffers assigned to the watchdog role of covering media "(AJR10). Étant donné la taille réduite du corpus, ces emplois restreints ne signifient pas que l'usage plus traditionnel n'existe plus, mais il est évident que le sens de watchdog a évolué avec le temps, probablement à la suite des réflexions sur l'éthique et les valeurs médiatiques, à l'époque où les organismes d'autorégulation et les rubriques « média » ont fait leur apparition.

Enfin, à la fin du dix-neuvième siècle, un autre terme fait son apparition : celui de beat (1875), signifiant le sujet précis couvert par un journaliste au sein d'une rubrique. L'apparition de ce terme marque une organisation plus précise et structurée au sein des rédactions, à l'époque où le journalisme devient un métier rémunéré et où les journaux recrutent du personnel. Ce terme a en réalité une origine policière, au sens de " $a$ regular route travelled by someone ", c'est-à-dire une ronde de police. Il ne s'agit pas d'un terme qui appartient exclusivement au domaine journalistique, ce qui souligne encore une fois le caractère peu spécifique des termes de ce domaine.

La période des journaux à un penny, qui prend fin vers 1890, voit ainsi l'émergence concomitante des enjeux commerciaux et de la valeur d'objectivité. Les termes créés à cette époque reflètent la formalisation des métiers et des genres dans les rédactions, ainsi que la définition plus précise du rôle de la presse dans la société. Au début du vingtième siècle, la notion d'objectivité est définie de façon plus précise encore à travers une réflexion sur les fonctions du journalisme.

\subsection{Formalisation du droit et codification des pratiques journalistiques}

Pendant la période qui s'étend de 1890 environ à 1970, les États-Unis connaissent de grandes réformes dans tous les domaines. Pour le journalisme, cette période se caractérise par une formalisation du droit, autant que le Premier Amendement peut le permettre, par l'intermédiaire de décisions de la Cour Suprême sur des sujets comme la diffamation, la protection de la vie privée ou la sécurité nationale. Parallèlement, les journaux et les associations professionnelles mènent une réflexion sur l'éthique de la profession, notamment lors de l'établissement de la commission Hutchins, qui voit le jour en 1942 dans le but de penser le rôle de la presse en démocratie. La période de 1890 à 1930 est également celle de l'apparition des muckrakers, premiers journalistes d'investigation qui, s'ils sont vivement critiqués pour leurs méthodes, n'en laissent pas moins une trace durable dans la conception de la profession.

À cette époque, de nouveaux genres émergent, plus précis que les précédents, notamment profile (1948) ou op-ed (1970). Ce dernier terme, dérivé d'editorial, fait référence à la page publiée en face de l'editorial (opposite the editorial), généralement consacrée à d'autres articles d'opinion. Cette diversification des genres est le signe d'une réflexion sur les sujets abordés ainsi que du développement de l'activité journalistique dans de nouvelles directions. Le genre du profil, par exemple, portraits approfondis de personnalités, représente une nouvelle manière d'aborder l'actualité pour les journalistes du milieu du vingtième siècle.

51 Suivant une logique de définition de plus en plus précise des textes produits, le terme de lead apparaît en 1912 pour désigner le premier paragraphe d'un article, lequel doit résumer l'essentiel des informations qu'il contient. Il faut noter que la première école 
de journalisme est créée en 1903 à l'Université Columbia, à l'initiative de Joseph Pulitzer. C'est à cette époque qu'est conduite une réflexion sur les techniques d'écriture, ce qui donne naissance à une certaine uniformisation du style journalistique. L'émergence du terme lead fait partie de ce mouvement. Dans le corpus, ce terme est peu employé pour désigner le premier paragraphe de l'article, probablement parce que les sujets des articles ne s'y prêtent pas, mais il prend plusieurs sens différents, par exemple: "Outside Missouri, newspapers leading with Ferguson also faced the question" (AJR3) ou "[...] rating its 100-year anniversary with an interesting choice for a lead story" (AJR4). Dans ces deux exemples, lead signifie tout ce qui est placé en première position : a lead story est l'article qui est placé en première page, et c'est également le sens retenu pour le verbe lead dans le premier exemple. Le sens de lead a évolué pour n'être plus restreint au premier paragraphe d'un article.

À cette période (1892), apparait également dans le domaine le terme story, qui fait référence à un article objectif et représente un hyperonyme pour tous les genres objectifs. Même s'il s'agit d'un mot très courant, sa redéfinition dans le domaine journalistique permet de le considérer comme un terme. Dans les écrits modernes, c'est le terme le plus fréquemment employé pour parler d'un article et c'est à cette époque qu'il se substitue à column, toujours suivant le principe de séparation de l'objectif et du subjectif. Il est employé de manière interchangeable avec un autre terme peu spécialisé, piece : "In the course of reporting my piece, some people speculated to me that [...] » (AJR2). Contrairement à l'idée selon laquelle un terme devrait avoir un sens précis, puisqu'il vise l'efficacité de la communication, le terme story a d'ailleurs une certaine plasticité sémantique. Ses emplois pour désigner un article dans le sens matériel du texte écrit sur le papier sont courants: "We'd done a disservice to the community by writing a story about Wilson at all » (EP9) ou «[the paper] gave the Ferguson story the banner »(AJR3). Cependant, il est également employé dans un sens plus large de contenu d'un article, comme dans l'expression courante get the story right : "We need leaders who aren't tempted to throw the first thing up on the Web just to get clicks, but want you to make sure you get the story right » (EP3). Enfin, ce terme est parfois simplement employé comme synonyme d'information, qui prend parfois un sens économique proche du sens de scoop, comme dans l'exemple suivant: "The journalists were accused of buying the story with gifts and favors" (EP9). Comme pour de nombreux termes du domaine, le sens de story ne semble pas fixé dans le discours des professionnels.

Enfin, c'est à partir de story qu'un autre terme définissant l'activité journalistique est créé : storytelling, le journaliste se définissant comme storyteller. C'est à la même époque, dans un effort de définition plus précise de l'activité journalistique, qu'apparaissent les termes de cover (1893) et coverage (1912), qui désignent le fondement de l'activité journalistique. Story, piece ou cover désignent des éléments fondamentaux de l'activité journalistique et prennent un sens particulier dans le domaine, mais ils apparaissent cependant comme peu complexes, compréhensibles en surface par le grand public, ce qui est sans doute révélateur de la même volonté d'employer un discours qui paraît peu technique.

54 Avec les réflexions sur les valeurs de la profession, d'autres termes apparaissent, reflétant une nouvelle conception de ce qui peut être publié. C'est ainsi qu'est créé un dérivé de report: reportable, désignant ce qui peut faire l'objet d'un travail journalistique. L'adjectif newsworthy apparaît en 1932, au sens des événements qui répondent aux critères de publication dans un journal. À l'inverse, le terme de tabloid 
est attesté comme type de journalisme à partir de 1917. À l'origine, ce terme signifie « $a$ compressed form or dose of anything "; c'est la petite taille des articles de ce type de journaux qui est à l'origine de ce terme.

Enfin, la réflexion sur les bonnes pratiques entraîne l'apparition de la locution adverbiale off the record. Cette notion devient l'une des conventions majeures de la profession : la plupart des documents éthiques des entreprises de presse insistent sur l'importance de respecter la confidentialité des propos tenus, lorsque celle-ci est explicitement demandée. Or, l'un des articles du corpus note l'incertitude terminologique de cette expression :

There are no set definitions for what off the record really means. As Mashable points out, "off the record" is "culturally binding, not legally binding. There is no official journalistic body that enforces the concept, or even defines it." (AJR6)

Même pour les acteurs du domaine, off-the-record peut être interprété comme une interdiction pure et simple de publier ce qui a été dit, ou bien comme une interdiction d'indiquer la source des propos, même lorsque ces derniers sont publiés (AJR6). Il s'agit donc d'un terme qui est mal défini dans la profession, ce qui facilite les écarts par rapport à la norme. Dans les médias numériques en particulier, de nombreux journalistes ne respectent pas ce principe, arguant que les personnes publiques ne devraient rien avoir à cacher et que les publications en ligne ont le droit de publier ce qu'elles jugent utile ou intéressant. De manière générale, l'usage massif des technologies numériques a engendré une redéfinition de certains aspects du domaine, processus qui se reflète dans la terminologie employée.

\subsection{L'ère numérique}

57 Depuis les années 1970, les journaux sont confrontés à des problèmes économiques nouveaux. De plus, depuis la fin des années 1990, l'apparition des médias numériques représente une nouvelle concurrence vers laquelle les annonceurs se dirigent de plus en plus, délaissant les journaux papier, qui manquent de ressources financières issues de la publicité. Ces modifications profondes du domaine ont eu un impact sur de nombreux aspects de la profession: les pratiques, les compétences numériques nouvelles nécessaires au métier ou l'éthique, comme nous l'avons signalé plus haut. Ces changements ont conduit à l'émergence de nouveaux termes.

De nombreux termes correspondant à la publication sur des plateformes numériques ont été intégrés au langage journalistique. De nouvelles plateformes de publication sont apparues, sur lesquelles le vocabulaire associé aux réseaux sociaux est très présent (social media, share counts, multi-channel and social media publishing, multi-platform publishing). Tous ces termes relèvent d'un nouveau type de journalisme, digital journalism, qui s'ajoute aux types de journalisme plus anciens également mentionnés dans le corpus: watchdog journalism, quality journalism, community journalism, big journalism. Il est intéressant de remarquer que les autres expressions font référence à des manières de rapporter l'actualité et de travailler, tandis que digital journalism se réfère uniquement à la plateforme de publication utilisée. Cela dit, le fait d'utiliser une plateforme numérique modifie de nombreux aspects des pratiques: le journaliste est souvent seul devant son ordinateur, l'article ne fait pas l'objet des mêmes corrections que dans une structure traditionnelle et, même lorsque la rédaction est plus importante, le temps de publication est plus court, le contenu est bien plus divers 
(vidéos, liens...) et les titres sont en général écrits pour attirer les clics. Il s'agit bien d'une modification profonde des pratiques qui justifie que digital journalism soit défini comme un type de journalisme à part entière, plutôt que comme un journalisme traditionnel publié sur une plateforme nouvelle.

De nouveaux termes reflètent les nouvelles préoccupations des entreprises de presse, et des journalistes, tels que track record ou click-based metrics. Il s'agit là de mesurer l'impact d'une publication en ligne sur les utilisateurs d'Internet. Un nouveau genre mentionné dans le corpus fait également son apparition : le listicle, article organisé sous forme de liste, en général numérotée. Comme les précédents, ce genre reflète l'angle sous lequel l'actualité est traitée, la particularité de celui-ci étant la forme courte, facilement lisible pour des utilisateurs d'Internet qui passent rapidement d'un site à l'autre.

60 Enfin, de nouveaux termes apparaissent pour définir l'activité journalistique telle qu'elle se déroule en ligne. Un article du corpus (AJR7) porte sur les robot reporters, ces machines capables de rédiger des articles, des machine-generated news, dans le cadre d'une nouvelle pratique appelée the automation of journalism. Cet article explique les équivalents entre les termes traditionnels décrivant l'activité journalistique et le robot writing ou algorithmic writing. Par exemple, l'activité appelée traditionnellement research est remplacée par content selection; selecting the information devient text planning; structuring est appelé sentence planning tandis que writing se transforme en linguistic realization.

Or, les termes correspondant à ce type de journalisme robotisé sont beaucoup plus techniques et complexes à comprendre que les termes employés en référence au journalisme traditionnel, ce qui n'est pas surprenant étant donné qu'ils appartiennent, à l'origine, au langage des programmeurs. Le fait que les équivalents soient donnés dans les articles montre bien que ces termes ne font pas encore partie du langage courant des journalistes. Cependant, ils y sont progressivement intégrés, tout comme l'ensemble des termes mentionnés dans cette section. Il semble que l'ère numérique ait entraîné une technicisation des termes autant que du métier. Au sein des écoles de journalisme, les formateurs insistent d'ailleurs sur les composantes techniques de la formation des jeunes professionnels, qui commencent dans le métier avec un ensemble de compétences en matière de numérique que leurs aînés ne possédaient pas (Peynaud 2013 : 260). Cette technicisation des termes accompagne logiquement l'évolution du métier et l'émergence de nouveaux besoins d'expression.

\section{Conclusion}

Ainsi, certains sous-domaines du journalisme ont fait l'objet de réflexions (comme celui des principes éthiques) ou d'une formalisation plus poussée (les genres, la division du travail), ce qui a donné lieu à l'émergence de termes pour les décrire. Il existe bien une terminologie employée dans la profession qui révèle une structuration conceptuelle partielle du domaine, contrairement à l'image peu spécialisée qui s'en dégage en première analyse.

63 Cependant, des pans entiers de la profession semblent ne pas avoir subi ce processus de formalisation. Notamment, ce qui concerne le cœur de l'activité journalistique, c'est-àdire la recherche d'informations ou l'écriture des articles, n'est pas décrit à l'aide de termes, mais avec des mots du langage courant. Comme le domaine de l'histoire, par 
exemple (Trouillon $2009: 25$ ), celui du journalisme n'emploie que peu de termes qui lui sont propres. Cela revient-il à dire que le domaine de la presse écrite aux États-Unis n'est pas réellement spécialisé ? En effet, Michel Van Der Yeught (2009: 33) explique que les premiers dictionnaires spécialisés, qui établissent les termes dans un domaine, sont le principal marqueur de l'émergence d'une langue de spécialité. Ici, la langue de spécialité du domaine ne semble pas entièrement structurée, certains sous-domaines ayant été laissés de côté par le processus historique d'émergence des termes.

Or, pour le cas du journalisme aux États-Unis, cette absence partielle de termes n'est pas fortuite : elle vient avant tout d'une spécificité culturelle, la volonté de représenter une activité ouverte à tous et de montrer que, comme le Premier Amendement l'impose, tous les citoyens sont des journalistes en puissance. Le choix d'employer un nombre réduit de termes dans certains sous-domaines, en ce sens, est l'un des éléments caractéristiques du domaine spécialisé du journalisme.

\section{BIBLIOGRAPHIE}

« About ». 2015. American Journalism Review, University of Maryland, consulté le 2 février 2015 $<$ http://ajr.org/about/>.

«About ». 2015. Editor and Publisher, Duncan McIntosh Company Inc., consulté le 2 février 2015 <http://www.editorandpublisher.com/About/>.

CABRÉ, Maria Teresa. 1998. La Terminologie, théories, méthodes et applications. Ottawa : Les Presses de l'Université d'Ottawa.

CANDEL, Danielle. 2004. « Wüster par lui-même ». In CORTÈs Colette. « Des fondements théoriques de la terminologie ». Cahiers du CIEL, Université Paris 7, 15-32.

DE CEGLIE, Audrey. 2007. « La communication organisationnelle au sein d'une entreprise de presse écrite ». Communication et organisation 31, Migrations conceptuelles [en ligne], consulté le 10 octobre $2012<\mathrm{http}$ //communicationorganisation.revues.org/204>.

HOFSTEDE, Gert Jan. 2005. Cultures and Organizations: software of the mind ( $2{ }^{\mathrm{e}}$ éd.). New York : McGraw-Hill.

Le Grand Dictionnaire Terminologique. [En ligne] Office québécois de la langue française. <http:// gdt.oqlf.gouv.qc.ca/index.aspx>.

Online Etymology Dictionary. <http://www.etymonline.com/index.php>.

PAdIolEAU, Jean-Gustave. 1985. Le Monde et le Washington Post. Paris : Presses Universitaires de France.

PeTIT, Michel. 2010. «Le discours spécialisé et le spécialisé du discours : repères pour l'analyse du discours en anglais de spécialité ». E-Rea 8.1, La Production et l'analyse des discours [en ligne], consulté le 12 novembre 2010 <http://erea.revues.org/1400>. 
PEYNAUD, Caroline. 2013. « Contribution à la caractérisation de la presse écrite américaine comme domaine spécialisé : milieu, culture et discours ». Thèse de doctorat en études anglaises. Université Paris Sorbonne.

RESCHE, Catherine. 2013. Economic Terms and Beyond: Capitalising on the Wealth of Notions. Linguistic Insights, vol. 176. Berne : Peter Lang.

RIEFFEL, Rémy. 2001. "Vers un journalisme mobile et polyvalent?». Quaderni 45 [en ligne], consulté le 9 octobre 2012

<http://www.persee.fr/web/revues/home/prescript/article /quad_0987-

1381_2001_num_45_1_1502>.

SAger, Juan C., David Dungworth \& Peter F. MCDONAlD. 1980. English Special Languages. Principles and Practice in Science and Technology. Wiesbaden : Brandstetter Verlag.

SCHUDSON, Michael. 1990. Origins of the Ideal of Objectivity in the Professions. New York: Garland Publishing.

TrouilLon, Jean-Louis. 2009. «Ébauche d'une caractérisation de l'anglais de l'histoire ». ASp 56, 527.

VAN DER YEUGHT, Michel. 2009. "Structurer l'anglais de la bourse : une approche des langues de spécialité par la spécialité ». Mémoire de synthèse d'Habilitation à diriger des recherches. Université Victor Segalen Bordeaux 2.

WATSON, John. 2008. Journalism Ethics by Court Decree, the Supreme Court on the Proper Practice of Journalism. New York : LFB Scholarly publishing.

\section{ANNEXES}

\section{Corpus}

\begin{tabular}{|c|c|}
\hline $\begin{array}{l}\text { Code de } \\
\text { l'article }\end{array}$ & Référence \\
\hline AJR1 & $\begin{array}{l}\text { LEVY, Dustin \& Katie TAKACS. 2014. « } 2 \text { Amusing Corrections and a Confession on } \\
\text { Common Mistakes ». American Journalism Review. Publié le } 12 \text { novembre } 2014 \text { [en ligne], } \\
\text { consulté le } 6 \text { février } 2015 \text { <http://ajr.org/2014/11/12/most-amusing-corrections-ever/ } \\
\text { >. }\end{array}$ \\
\hline AJR2 & $\begin{array}{l}\text { LEVY, Dustin. 2014. "Vice, and the Trend of First-Person Journalism ». American } \\
\text { Journalism Review. Publié le } 1^{\text {er }} \text { décembre } 2014 \text { [en ligne], consulté le } 6 \text { février } 2015 \\
\text { <http://ajr.org/2014/12/01/vice-trend-first-person-journalism/>. }\end{array}$ \\
\hline AJR3 & $\begin{array}{l}\text { LOGUE, Josh. 2014. «Front Pages after the Ferguson 'No Indictment' Decision ». American } \\
\text { Journalism Review. Publié le } 25 \text { novembre } 2014 \text { [en ligne], consulté le } 6 \text { février } 2015 \text { < } \\
\text { http://ajr.org/2014/11/25/front-pages-ferguson-no-indictment-decision/>. }\end{array}$ \\
\hline AJR4 & $\begin{array}{l}\text { CREASMAN WELCOME, Rose. 2014. «Glass Still 'Shattered,' } 16 \text { Years Later ». American } \\
\text { Journalism Review. Publié le } 12 \text { novembre } 2014 \text { [en ligne], consulté le } 6 \text { février } 2015 \\
\text { <http://ajr.org/2014/11/12/stephen-glass-new-republic-anniversary/>. }\end{array}$ \\
\hline
\end{tabular}




\begin{tabular}{|c|c|}
\hline AJR5 & $\begin{array}{l}\text { SINGER, Dan. 2014. «Music Critics See Their Role and Influence Waning in The Era of } \\
\text { Digital Music ». American Journalism Review. Publié le } 13 \text { novembre } 2014 \text { [en ligne], } \\
\text { consulté le } 6 \text { février } 2015<\text { http://ajr.org/2014/11/13/music-critics-role-changing/>. }\end{array}$ \\
\hline AJR6 & $\begin{array}{l}\text { BLAIR, Cory. 2014. «Off the record, but online ». American Journalism Review. Publié le } 28 \\
\text { novembre } 2014 \text { [en ligne], consulté le } 6 \text { février } 2015 \text { <http://ajr.org/2014/11/28/off- } \\
\text { record-online/>. }\end{array}$ \\
\hline AJR7 & $\begin{array}{l}\text { Contributing writer. 2014. « Robot Reporters or Human Journalists: Who Do You Trust } \\
\text { More? ». American Journalism Review. Publié le } 24 \text { octobre } 2014 \text { [en ligne], consulté le } 6 \\
\text { février } 2015 \text { <http://ajr.org/2014/10/24/who-trusts-robot-reporters/>. }\end{array}$ \\
\hline AJR8 & $\begin{array}{l}\text { SHASTRY, Anjali. 2015. "Rumors, Lies and the Internet: } 7 \text { Questions for Craig } \\
\text { Silverman ». American Journalism Review. Publié le } 26 \text { novembre } 2014 \text { [en ligne], consulté } \\
\text { le } 6 \text { février } 2015 \text { <http://ajr.org/2014/11/26/rumors-lies-internet-7-questions-craig- } \\
\text { silverman/>. }\end{array}$ \\
\hline AJR9 & $\begin{array}{l}\text { KOMUVES, Anita. 2014. «Sputnik: Russian Propaganda With Some Fluff ». American } \\
\text { Journalism Review. Publié le } 11 \text { novembre } 2014 \text { [en ligne], consulté le } 6 \text { février } 2015 \\
\text { <http://ajr.org/2014/11/11/propaganda-fluff/>. }\end{array}$ \\
\hline AJR10 & $\begin{array}{l}\text { TAKACS, Katie. 2014. « That Awkward Moment When Journalists Must Write About Their } \\
\text { Employer ». American Journalism Review. Publié le } 3 \text { novembre } 2014 \text { [en ligne], consulté le } \\
6 \text { février } 2015 \text { <http://ajr.org/2014/11/03/awkward-moment-journalists-must-write/>. }\end{array}$ \\
\hline EP1 & $\begin{array}{l}\text { PECK, Gretchen. 2014. "An Engaging Proposal ». Editor and Publisher. Publié le } 3 \\
\text { novembre } 2014 \quad \text { en ligne], consulté le } 6 \text { février } 2015 \text { <http:// } \\
\text { www.editorandpublisher.com/Features/Article/An-Engaging-Proposal>. }\end{array}$ \\
\hline EP2 & $\begin{array}{l}\text { YANG, Nu. 2015. «Calling all newspapers ». Editor and Publisher. Publié le } 5 \text { janvier } 2015 \\
\text { [en ligne], consulté le } 6 \text { février } 2015<\text { http://www.editorandpublisher.com/Features/ } \\
\text { Article/Calling-All-Newspapers>. }\end{array}$ \\
\hline EP3 & $\begin{array}{l}\text { PECK, Gretchen. 2015. « Blending the Best of Old and New ». Editor and Publisher. Publié le } \\
2 \text { février } 2015 \quad \text { [en ligne], consulté le } 6 \text { février } 2015 \text { <http:// } \\
\text { www.editorandpublisher.com/Features/Article/Blending-the-Best-of-old-and-New>. }\end{array}$ \\
\hline EP4 & $\begin{array}{l}\text { PECK, Gretchen. 2014. « Digital Printing Strategies for 2015: What's New, What's Next? ». } \\
\text { Editor and Publisher. Publié le } 8 \text { décembre } 2014 \text { [en ligne], consulté le } 6 \text { février } 2015 \\
\text { <http://www.editorandpublisher.com/Features/Article/Digital-Printing-Strategies- } \\
\text { for-2015--What-s-New--What-s-Next->. }\end{array}$ \\
\hline EP5 & $\begin{array}{l}\text { YANG, Nu. 2014. «E\&P's } 2014 \text { Publisher of the Year: Karen Andreas, North of Boston } \\
\text { Media Group ». Editor and Publisher. Publié le } 30 \text { octobre } 2014 \text { [en ligne], consulté le } 6 \\
\text { février } 2015 \quad \text { <http://www.editorandpublisher.com/Features/Article/E-P-s-2014- } \\
\text { Publisher-of-the-Year--Karen-Andreas--North-of-Boston-Media-Group>. }\end{array}$ \\
\hline EP6 & $\begin{array}{l}\text { SCHULT, Eric. 2015. "Production: User Groups Adapt to New Realities ». Editor and } \\
\text { Publisher. Publié le } 20 \text { janvier } 2015 \text { [en ligne], consulté le } 6 \text { février } 2015 \\
\text { <http://www.editorandpublisher.com/Features/Article/Production--User-Groups- } \\
\text { Adapt-to-New-Realities>. }\end{array}$ \\
\hline
\end{tabular}




\begin{tabular}{|c|c|}
\hline EP7 & $\begin{array}{l}\text { PECK, Gretchen. 2014. "Refuse to be Silenced». Editor and Publisher. Publié le } 8 \\
\text { septembre } 2014 \quad \text { [en ligne], consulté le } 6 \text { février } 2015 \text { <http:// } \\
\text { www.editorandpublisher.com/Features/Article/Refuse-to-be-Silenced>. }\end{array}$ \\
\hline EP8 & $\begin{array}{l}\text { YANG, Nu. 2014. «The right stuff». Editor and Publisher. Publié le } 20 \text { octobre } 2014 \text { [en } \\
\text { ligne], consulté le } 6 \text { février } 2015 \text { <http://www.editorandpublisher.com/Features/ } \\
\text { Article/The-Right-Stuff>. }\end{array}$ \\
\hline EP9 & $\begin{array}{l}\text { PECK, Gretchen. 2014. «Trust in Media ». Editor and Publisher. Publié le } 6 \text { octobre } 2014 \\
\text { [en ligne], consulté le } 6 \text { février } 2015 \text { <http://www.editorandpublisher.com/Features/ } \\
\text { Article/Trust-in-Media>. }\end{array}$ \\
\hline EP10 & $\begin{array}{l}\text { KANE, Rich. 2014. « Weathering the storm ». Editor and Publisher. Publié le } 10 \text { novembre } \\
2014 \text { [en ligne], consulté le } 6 \text { février } 2015 \text { <http://www.editorandpublisher.com/ } \\
\text { Features/Article/Weathering-the-Storm>. }\end{array}$ \\
\hline
\end{tabular}

\section{NOTES}

1. Nous considérons le journalisme dans son ensemble comme une profession structurée en savoir-faire abstraits et enseignés à l'université. Cette profession est divisée en métiers qui incluent éditeur, correcteur, journaliste économique, etc.

2. Les références des articles du corpus sont données en annexe.

3. Le Grand Dictionnaire Terminologique. [en ligne] Office québécois de la langue française. <http:// gdt.oqlf.gouv.qc.ca/index.aspx>.

4. Ce dictionnaire en ligne est principalement une compilation de quatre ouvrages d'étymologie : Oxford English Dictionary (second edition), Barnhart Dictionary of Etymology (1988), Etymological Dictionary of Modern English (1921), Comprehensive Etymological Dictionary of the English Language (1971). L'utilisation de ce site plutôt que d'un seul de ces ouvrages permet de varier les sources pour obtenir une image plus complète.

5. Nous parlons d'article « objectif » pour reprendre la distinction opérée par les professionnels eux-mêmes, tout en reconnaissant que tout texte a nécessairement une dimension subjective. Les articles dits «subjectifs» se distinguent cependant par l'expression explicite d'un point de vue, ce qui n'est pas le cas des articles dits "objectifs». La notion d'objectivité doit être comprise davantage comme un but à atteindre que comme une description factuelle.

\section{RÉSUMÉS}

Pour le linguiste, les termes employés dans un domaine spécialisé, pour la communication entre spécialistes, représentent une porte d'entrée vers la culture professionnelle. L'intégration d'une analyse terminologique et d'une approche culturelle donne des indications précieuses sur le fonctionnement d'une communauté professionnelle. Cette approche interdisciplinaire est particulièrement utile pour le domaine spécialisé des journalistes de presse écrite aux États-Unis dans la mesure où leur culture professionnelle est difficile à définir pour un observateur extérieur. Ainsi, à partir d'un corpus composé d'articles de revues professionnelles, il est possible 
de dresser un tableau de certains éléments significatifs de cette culture tels qu'ils apparaissent à travers les termes. Dans le discours des journalistes de presse écrite, ce métier apparaît notamment comme très peu spécialisé et orienté vers la technique plus que vers la théorie. Historiquement, cette image correspond au développement de la profession et à la formalisation progressive de certains aspects du métier, tandis que d'autres restent peu spécialisés.

For a linguist, the terms used in a specialized domain for internal communication represent entry points to explore its professional culture. By combining an analysis of terminology with a cultural approach, much valuable information can be found on the way a professional community functions. Such an interdisciplinary approach is especially useful to understand the specialized domain of written-press journalists in the United States as their professional culture is difficult to define for an outside observer. Thus, analyzing a corpus composed of professional articles makes it possible to identify some significant elements of this culture as they appear through the terms. The results show that written-press journalism is only slightly specialized and more oriented towards technique rather than theory. Historically, this representation corresponds to the development of the profession and the gradual formalization of some aspects only, while others remain relatively unspecialized.

\section{INDEX}

Mots-clés : culture professionnelle, journalisme, spécialisation, terminologie, variété spécialisée de l'anglais

Keywords : professional culture, journalism, specialization, specialized variety of English, terminology

\section{AUTEUR}

\section{CAROLINE PEYNAUD}

Caroline Peynaud est maître de conférences en langue de spécialité en LEA à l'Université Grenoble Alpes depuis 2014. Elle s'intéresse à l'analyse de la notion de spécialisation dans la presse généraliste de qualité aux États-Unis. Elle est membre du GREMUTS au sein du laboratoire ILCEA4. caroline.peynaud@u-grenoble3.fr 